\title{
Von resonanten Subjekten und responsiven Strukturen
}

\author{
Eine Auseinandersetzung mit Hartmut Rosas Soziologie \\ der Weltbeziehungen aus Sicht des Critical Realism
}

Lisa Waldenburger und Hannes Teutoburg-Weiss

\section{EinLeitung}

Kurze Zeit nach dem die Grundwidersprüche der kapitalistischen Gesellschaftsformation in der Finanzkrise $2007 \mathrm{zu}$ Tage getreten sind, verpflichteten sich Hartmut Rosa und zwei seiner Jenaer Kollegen auf einen »große[n] Akt der Erneuerung: die Rückkehr der Kritik in die Soziologie« (Dörre/Lessenich/Rosa 2009: 12). Die von Rosa (2016) mittlerweile vorgelegte Soziologie der Weltbeziehungen kann als ein Baustein eines solchen Aktes gelten, kulminiert sie doch in der Kritik der Resonanzverhältnisse oder vielmehr in der Kritik der Entfremdungsverhältnisse der (Spät-)Moderne. ${ }^{1}$ In Zeiten, in denen sich die Kritische Theorie in einem circulus vitiosus befindet und fortwährend über die Möglichkeitsbedingungen von sozialwissenschaftlicher Kritik streitet oder postmoderne Philosophien das Aufzeigen von Kontingenz mit substantieller Sozialkritik verwechseln, sind Rosas Ausführungen begrüßenswert anregend: Entfremdete Selbst-Welt-Verhältnisse sind das Problem; resonante Beziehungen die mögliche Lösung; die Analyse und Kritik der gegenwärtigen Formen der Vergesellschaftung der erste wichtige Schritt.

Allein aus einem solchen Baustein heraus lässt sich allerdings noch kein Gewölbe errichten, in dem eine zeitgemäße, kritische Sozialwissenschaft beherbergt sein kann. Es scheint demnach unumgänglich den angesprochenen

1 | Peter Schulz weist pointiert und schlüssig darauf hin, dass sich der Bezugspunkt von Rosas Kritik in seiner Theorieentwicklung von einer genuin kapitalismuskritischen Betrachtung hin zu einer breiteren Modernekritik verschoben hat (vgl. Schulz 2015). Mit seiner Resonanztheorie ist er endgültig bei Letzterem angekommen. 
Erneuerungsakt als multidisziplinäres Gemeinschaftsprojekt zu verstehen. Wenn Rosa gewissermaßen die entsprechende Soziologie zu liefern vermag, könnte der Critical Realism (CR) - dem ein ähnlich emanzipativer Impetus innewohnt - eine philosophische und sozialtheoretische Grundlegung beisteuern. Roy Bhaskar schreibt dem CR in Hinblick auf wissenschaftliche Untersuchungen dabei explizit die Rolle einer Hebamme (midwife) oder einer Hilfsarbeiterin (underlabourer) zu (vgl. Bhaskar 2008: 254).

Wichtig ist bei der Überprüfung der Kompatibilität der beiden Perspektiven aber in erster Linie nicht allein die Kongruenz ihrer normativen Anker, sondern die Vereinbarkeit ihrer anthropologischen und sozialtheoretischen Grundannahmen. Andrew R. Sayer führt zu Recht an, dass solche Annahmen $z u$ jeder Zeit in jeglicher sozialwissenschaftlichen Tätigkeit gemacht werden:

"It's impossible to avoid making assumptions about human nature in social science even those who believe we are purely socially or culturally determined presuppose that we are susceptible to such determination - so it's better to make these assumptions explicit, rather than risk leaving them unexamined. We need a philosophical anthropology - not one that posits universal uniformities to the exclusion of difference, but one that identifies commonalities and sources of difference." (Sayer 2011: 98-99)

Wir teilen an dieser Stelle Sayers Argument für die Notwendigkeit einer ausformulierten Anthropologie (und Sozialtheorie) und machen dies zur Ausgangsüberlegung unseres Beitrages. Nachfolgend soll dem Passungsverhältnis von Rosas Soziologie der Weltbeziehungen mit der Sozialtheorie des CR nachgespürt werden. Im Rahmen dieser Problemstellung rekurriert der Beitrag kaum auf Rosas Gesellschaftstheorie oder Zeitdiagnose, sondern versucht vielmehr die stellenweise nur implizit vorhandenen und dünn gestreuten Grundannahmen über Subjekt, Welt und deren Verhältnis mit denen des CR zu kontrastieren. Wir vertreten dabei die These, dass vor allem der Begriff der Weltbeziehung einen äußerst fruchtbaren Beitrag für eine zeitgemäße Sozialtheorie darstellt. Ambivalenter ist unsere Einschätzung bezüglich der analytischen Relevanz der Resonanz- und Entfremdungskategorien.

Der Beitrag untergliedert sich in zwei Hauptteile: Im Ersten wird Rosas eigener Anspruch hinsichtlich einer Soziologie der Weltbeziehungen, welche die Beziehungsidee radikalisieren soll, besprochen und mit dem Praxis-Konzept des CR in Beziehung gesetzt. Im zweiten Teil soll überprüft werden, ob Rosas theoretische Ausführungen zu menschlicher Subjektivität im Rahmen von Margaret Archers Subjektkonzeption anschlussfähig sind. 


\section{Die Selbst-Welt-Beziehung - Eine Radikalisierung der Beziehungsidee?}

Zugleich Ausgangspunkt und ausweisbare Stärke von Rosas Erneuerungsbeitrag ist die Hinführung heterogener, aus Philosophie, Soziologie und Naturwissenschaft stammender Theoriefragmente auf einen Fokalpunkt: Eine Soziologie der Weltbeziehungen. Selbst wenn Rosa nachdrücklich betont, dass es ihm »entscheidend darauf ankommt, ihre [die der Weltbeziehungen] gesellschaftliche Prägung und damit auch ihre Variabilität und Veränderbarkeit zu untersuchen und herauszustellen « (Rosa 2016: 70, A.d.V.), ${ }^{2}$ so gründet sich seine Untersuchung dennoch in Evidenzen, Annahmen und Spekulationen über die conditio humana und die Verfasstheit der Welt, welche er aus eben diesen unterschiedlichen Bezugspunkten herausdestilliert (vgl. ebd.: Teil I). Als anthropologisches Grundpostulat kann dabei die immerwährende Bezogenheit des Subjekts auf die es umschließende Welt gelten.

"Subjekte stehen der Welt also nicht gegenüber, sondern sie finden sich immer schon in einer Welt, mit der sie verknüpft und verwoben sind, der gegenüber sie je nach historischem und kulturellem Kontext fließende oder auch feste Grenzen haben, die sie fürchten oder lieben, in die sie sich geworfen oder in der sie sich getragen fühlen etc." (Ebd.: 63)

Mit der Setzung einer formal ahistorischen Selbst-Welt-Bezogenheit postuliert Rosa gleichzeitig eine Radikalisierung der Beziehungsidee. Diese Radikalisierung ruft ein Grundproblem der bisherigen Sozialtheorie- bzw. Philosophiegeschichte in Erinnerung und soll es im selben Augenblick überwinden: Das konzeptionelle Problem der analytischen Trennung und Bestimmung von Subjekt und (sozialer) Welt (vgl. ebd.: 61). In Rosas konkreter Antwort begegnen Subjekt und Welt dann einander nicht mehr als vorbestimmte Entitäten, sondern ihre Haupteigenschaft besteht in der wechselseitigen Formbarkeit während gegenseitiger Bezugnahme (vgl. ebd.: 62-69).

Nun lassen sich innerhalb Rosas Soziologie der Weltbeziehungen aber mindestens zwei unterschiedliche Betonungen dieser Radikalisierung der Beziehungsidee aufspüren. In der strikten Auslegung vollzieht Rosa einen Schulterschluss mit Bruno Latour (1991, 2005) als Vertreter einer Akteur-Netzwerk-Theorie. Entitäten besitzen dort keine abgrenzbare Qualität, vielmehr erscheinen sie als Knotenpunkte in heterogenen Netzwerken, die ihrerseits sowohl menschliche als auch nicht-menschliche Aktanten einschließen. Diese Aktanten erhalten ihre konkreten Eigenschaften jedoch erst innerhalb des Konstitutionsprozesses eines bestimmten Netzwerkes. Auch wenn Rosa

2 | Siehe hierzu auch Rosa 2016: 753. 
vorrangig nicht der Netzwerkmetaphorik folgt, sondern an deren Stelle das Subjekt-Welt-Verhältnis stark macht, gibt es gerade in dem expliziter sozialtheoretischen Teil seines Entwurfes Fundstellen, die einer relationalen Ereignisontologie - wie sie u.a. Latour vertritt - sehr nahekommen. ${ }^{3}$

Aus Sicht des CR ist eine solche Grundlegung jedoch nicht haltbar, weil sie mit einer flachen Ontologie operiert und damit die Verfasstheit der Welt - als abgestuft stratifizierte Welt, in der Dinge zwar in Relationen stehen, sich aber niemals in diesen erschöpfen - nicht begreifen kann (vgl. Elder-Vass 2008: 465). Es ist danach unzulässig - wie die Resonanztheorie in dieser Auslegung es tut - von (beobachtbaren) Ereignissen auf strukturelle Eigenschaften von Dingen/Entitäten/Aktanten zu schließen (vgl. zur Kritik eines solchen Schlusses: ebd.: 465f.). Ebenso problematisch erscheint die theoretische Nivellierung der Mensch-Ding-Differenzierung sowie der Natur-Kultur-Unterscheidung. Auch wenn der CR eine prinzipiell ablehnende Position zum Natur-Kultur-Dualismus einnimmt, da Kultur immer als Teil von Welt begriffen werden sollte (vgl. Lindner 2014: 218), muss festgehalten werden, dass natürliche Strukturen im Gegensatz zu ihrem sozialen Pendant andere Eigenschaften besitzen und nicht auf die Reproduktion durch menschliches Handeln angewiesen sind (vgl. Bhaskar 1998: 42). Dies heißt im Umkehrschluss jedoch nicht, dass menschliches Handeln in Bezug auf natürliche (Um-)Welt konsequenzlos ist. ${ }^{4}$

Über diese Fragen der ontologischen Basisannahmen hinaus, würde eine solch strikte Auslegung weitergehende Konsistenzprobleme für eine Soziologie der Weltbeziehungen im Sinne Rosas mit sich bringen:

3 | Vgl. bspw.: "[B]eide Seiten - Subjekt und Welt - [werden] in der und durch die wechselseitige Bezogenheit erst geformt, geprägt, ja mehr noch: konstituiert [...]." (Rosa 2016: 62, A./Herv. d.V.) oder auch die affirmative Besprechung Latours symmetrischer Anthropologie (vgl. ebd.: 384f.).

4 | Ein verwandtes Problem findet sich in der Auslegung von Formulierungen, in denen die Welt von Rosa personifiziert wird. Ist dies reine Metaphorik oder geht Rosa wie zum Beispiel Michel Callon (1984) oder Latour (1996) von einer Artikulationsfähigkeit der nicht-menschlichen Welt aus? Dave Elder-Vass führt zu dieser Problematik der unterschiedlichen Eigenschaften von Dingen und ihrer kausalen Wirkmächtigkeit in Bezug zur Akteur-Netzwerk-Theorie aus: "For critical realists, scallops, motors, and other non-human objects are significant in sociological explanations because they have causal powers - just as human agents are significant in sociological explanations because they have causal powers. But scallops have different causal powers from humans, and different causal powers from motors. [...] We achieve symmetry in the treatment of human and non-human actors, not by treating them all in the same terms, but by treating each in the terms that are appropriate to its own particular structure and properties" (EIder-Vass 2008: 469 Herv. i.0). 
1. Biographische Historizität und Ausformung einer halbwegs geschlossenen Identität lassen sich nicht denken, weil es kein subjektives Narrativ gibt, welches außerhalb von externen Beziehungsprozessen steht.

2. Weiterführend: Die für die Kritische Theorie und auch Rosa so zentrale Kategorie der subjektiven Leid-Erfahrung - u.a. besprochen als Entfremdung - ist in der Theorietradition der Ereignisontologien unverständlich, weil es keine Differenz zwischen momentan subjektiver Interaktion mit Welt und nicht-situativ, intrinsisch leidendem Selbst geben kann.

3. Welt hat in dieser hochdynamischen Perspektive keine Historizität, da momenthafter Veränderung gegenüber dem andauernd Bestehenden eine signifikant höhere Bedeutung zukommt.

Am Ende wird die Analyse und Kritik von (strukturellen) Möglichkeitsbedingungen subjektiver Handlungs-/Resonanzfähigkeit in einer solchen Perspektive gegenstands-, ziel- und sinnlos.

Wir plädieren an dieser Stelle aufgrund der angeführten Aspekte für eine schwächere Auslegung der Radikalisierung der Beziehungsidee. Diese bricht sich vor allem in den beispielhaften Ausführungen und Erklärungen Rosas Bahn: Subjekte und soziale Welt gehen hier nicht aus jeder Beziehung neu hervor, sondern sie sind füreinander lediglich ko-konstitutiv und erst in ihrem Begegnen wandeln sich potentiell beide. ${ }^{5}$ Diese Auslegung wäre kompatibel mit der sozialtheoretischen Position und Tiefenontologie des CR. Welt und Selbst werden demnach nicht wechselseitig innerhalb eines spezifischen Verhältnisses konstruiert, sondern aufgrund eines Verhältnisses lagern sich neue Sedimente auf den bereits versteinerten Schichten der einzelnen Beziehungspartner ab oder es werden ältere Schichten abgetragen. Das Subjekt wird zum kognitiven Bastler, dessen Materialien von der Welt strukturell vorgegeben sind (vgl. Bhaskar 1998: 37). Subjekt und Welt erhalten damit gegenüber der strikten Auslegung eine gemeinsame Geschichte, die durch ihre jeweilig unterschiedlichen Eigenschaften mitgeschrieben wird. Diese Perspektive setzt einerseits Entitäten voraus, die sich aufeinander beziehen können - mit ihren jeweils spezifischen Vermögen und Eigenschaften. Andererseits wird aber der gegen-

5 | Für eine solche Auslegung sprechen beispielsweise Teile der Resonanz-Definition "Resonanzbeziehungen setzen voraus, dass Subjekt und Welt hinreichend igeschlossen, bzw. konsistent sind, um mit je eigener Stimme zu sprechen und offen genug um sich affizieren oder erreichen zu lassen " (Rosa 2016: 298, Herv. d.V.). Oder auch: "[...] Subjekte wie Welt [sind] in ihrer konkret gegebenen und erfahrbaren Gestalt immer schon das Ergebnis vorgängiger Beziehungsverhältnisse oder Relationen [...]" (ebd.: 289, A./Herv. d.V.). 
seitigen Abhängig- und Wandelbarkeit Rechnung getragen. ${ }^{6}$ Mit anderen Worten ist hier im Sinne des CR von einer Scheidung zu sprechen, die auf einer ontologischen Differenz fußt:

"Society produces us as the people that we are, 'out of biologically given raw material, and it continues to transform us throughout our lives. We in turn make new societies out of old societies by our actions, whether intentionally or not and to whatever extent the new society either replicates the old one or is radically different." (Collier 1994: 144f.)

Für die soziale Welt wird die weiter oben angesprochene, konzeptionelle Problematik bei Bhaskar (1998) und Archer (1995) explizit diskutiert und in einer doppelten Dualität aufgelöst: Soziale Strukturen sind gleichzeitig Voraussetzung und Ergebnis von Praxis; Praxis ist der ereignishaft erscheinende Vollzug der Vermittlung von structure and agency; dieser Vollzug schließt die bewusst intentionale Produktion sowie die meist unbewusste Reproduktion seiner eigenen Bedingungen mit ein (vgl. Bhaskar 1983: 84; 2009: 125-35; Archer 1995: 71-80). ${ }^{7}$ Strukturen und Akteure haben aber bereits spezifische Eigenschaften außerhalb einer konkreten Praxisbeziehung und sind damit vor dieser ontologisch unterscheidbar: Strukturen sind gegenüber handelnden Subjekten emergent. Beide werden jedoch erst in Praxis aufeinander bezogen und sozusagen >artikulieren< sich erst dann ihre Eigenschaften. In der Konsequenz lassen sich mit der Sozialontologie des CR beidseitig transformative und reproduzierende Praxen gleichermaßen adäquat denken.

Wie bereits umrissen kommt dem Praxisbegriff in der Sozialtheorie des CR eine zentrale Bedeutung zu, weil erst in ihm deutlich wird, dass Gesellschaft sich nur aktiv - das heißt durch handelnde Subjekte - reproduzieren kann und dass strukturelle Ermöglichungen und Einschränkungen für die Subjekte erst in eben dieser Auseinandersetzung mit sozialer Wirklichkeit deutlich werden

6 | Die potentiell offene Frage nach einer ursprünglichen Beziehung ist in einer (Sozial-)Ontologie, welche die Emergenz von Entitäten und ihre strukturellen Eigenschaften betont, geradezu nichtig. Die spezifischen Eigenschaften einer Entität lassen sich eben nicht allein durch die Summe ihrer in Relation stehenden Teile erklären.

7 | Bhaskar verdeutlicht diese konstitutive Bezogenheit von Selbst und sozialen Strukturen - entspricht der sozialen Welt bei Rosa - im Rahmen seines Transformational Model of Social Activity (vgl. Bhaskar 1998: Kap. 2). Archer greift diesen Ansatz auf und entwickelt inn in als Morphogenetic Approach weiter (vgl. Archer 1995: 137-160). Beide Versuche einer sozialontologischen Theoretisierung können als Antwort auf das von Rosa aufgeworfene konzeptionelle Grundproblem des Selbst-Welt-Verhältnisses gelesen werden. Ebenso wie Rosa lehnen Bhaskar und Archer sowohl einen methodologischen Individualismus als auch einen methodologischen Holismus ab (vgl. Bhaskar 1998: 33-35; Archer 1995: 81-89). 
(vgl. Bhaskar 1998: 39-45; Archer 1995: 195-200). Anders ausgedrückt ist Praxis hier als Beziehungskategorie unhintergehbar. Hinsichtlich des Begriffspaares Weltbeziehung (Rosa) und Praxis (CR) kann man im Ergebnis eine gewisse konzeptionelle Kompatibilität konstatieren.

Problematisch erscheint im Falle des CR lediglich die Tendenz der (Über-) Betonung von aktiv intentionaler Gestaltung gegenüber >passiver Welteingebundenheit auf Subjekt-Seite. Rosa leistet hier einen aus unserer Sicht sozialtheoretisch wichtigen und vor allem anschlussfähigen Beitrag, indem er die Bezogenheit des Subjektes auf (soziale) Welt nicht von vorneherein als aktive Tätigkeit konzipiert. ${ }^{8}$ Eine Weltbeziehung ist demnach

"[...] die Art und Weise, wie Menschen in die Welt gestellt sind oder, besser: in der sie sich als in die Welt gestellt erfahren. Diese Stellung oder auch Haltung der Welt gegenüber umfasst sowohl die eher passive Seite der Welterfahrung als auch die aktive Weise des menschlichen Eingreifens in die Welt [...]." (Rosa 2012: 7)

Führt man die umrissenen Gedanken allerdings zu ihrem Endpunkt, ergibt sich mit dieser weicheren Auslegung ein neues konzeptionelles Problem für Rosa bezüglich der resonanten, respektiven stummen Beziehung zwischen Welt und Subjekt. Der CR geht davon aus, dass soziale Welt und Subjekt zwangsläufig in einer Beziehung stehen müssen, welche geradezu konstitutiv responsiv ist, weil jede Form von Praxis-Struktur reproduzierend respektive transformierend ist und damit die Bedingungen zukünftiger, subjektiver Handlungsfähigkeit durch sie erst gesichert werden. Es gibt analytisch auf dieser Ebene keine Form der Nicht-Responsivität. Das individuelle Handeln hat mit jedem ausgeführten Akt Konsequenzen. Wäre es nicht so, dann besäße das Subjekt keine Agency.

Die relevante Frage ist vielmehr, ob das Subjekt diese Responsivität auch als solche wahrnimmt. Der einzige Moment, in dem man im Rahmen des CR von Resonanz als respektiver Entfremdung sprechen kann, wäre eine evaluative Dimension von Praxis (als Ereignis) qua Erfahrung oder Reflexion durch das Subjekt selbst. Damit bekommt das Resonanz- und Entfremdungskonzept allerdings eine subjektive Schlagrichtung, die Rosa bei seiner Besprechung von Erich Fromm ausdrücklich zurückweist (vgl. Rosa 2016: 565-573).

Allerdings scheint Rosa an anderer Stelle bezeichnenderweise einen eben solchen Perspektivenwechsel zu vollziehen. Rekurriert die Resonanzdefinition noch auf eine gegenseitige Antwortbeziehung: »Resonanz ist keine Echo-,

8 Instruktiv ist an dieser Stelle die innerhalb des CR entbrannte Debatte hinsichtlich des Stellenwertes von Habitus gegenüber Reflexivität in der Subjektkonzeption Archers. Zur Habitus versus Reflexivitäts-Debatte vgl. auch Elder-Vass 2007, Fleetwood 2008, Sayer 2010 sowie Archer 2010. 
sondern eine Antwortbeziehung; sie setzt voraus, dass beide Seiten mit eigener Stimme sprechen« (ebd.: 298, Herv. i.O.), sodass sich Indizien für eine resonante Beziehung auf beiden Seiten finden lassen müssen, findet sich bei der Entfremdungsdefinition nur noch die subjektive Erfahrung: »Entfremdung definiert damit einen Zustand, in dem die >Weltanverwandlung « misslingt, so dass die Welt stets kalt, starr, abweisend und nichtresponsiv erscheint « (ebd.: 316, Herv. d.V.). Mit anderen Worten wird aus der vormaligen Beschreibung einer objektivierbaren Subjekt-Welt-Beziehung (Resonanz) zwischen den Definitionen die Explikation einer subjektiven Wertung, welche über die wahrgenommene Beziehung richtet (Entfremdung).

Erscheinen aus dieser ersten Perspektive des CR der Resonanz- und Entfremdungsbegriff als Beziehungsbegriffe analytisch redundant, so könnten sie womöglich ihre Potentiale dort entfalten, wo sie erfahrend respektive evaluativ gewendet in die Subjektkonzeption einfließen. Dies soll, neben dem Abgleich anderer Konzepte der subjektiven Seite der Weltbeziehung, nachfolgend überprüft werden.

\section{Die Subjektseite der Weltbeziehungen - EINE SUbJeKtive Wendung Des Resonanzbegriffs?}

Neben der (sozial-)ontologischen Unterscheidung von Mechanismen, Ereignissen und dem Erfahrbaren sowie der sozialtheoretischen Fundierung des structure-and-agency-Problems mittels des Transformational Model of Social Activity und des Morphogenetic Approach, ist die Subjekttheorie von Archer einer der wichtigsten Bestandteile des CR (vgl. Lindner 2014: 231). An anderer Stelle bereits als Konzeption einer situierten Reflexivität eingeführt (vgl. Waldenburger 2014), soll diese Subjekttheorie im Folgenden entlang ihrer Grundzüge dargestellt und mit dem von Rosa, im Rahmen seiner Resonanztheorie implizit entwickelten Verständnis von Subjektivität, ins Verhältnis gesetzt werden.

Das kritisch-realistische Subjektverständnis Archers geht von einem stratifizierten Selbst aus, welches sich über vier Ebenen erstreckt: (1) Selbst und Welt, (2) soziale Identität, (3) innere Konversation und (4) Selbst als komplexes Ganzheitliches. Anhand der zwangsläufigen Verortung des Subjekts innerhalb von Welt, verfügt es nach Archer über grundlegende, personale emergente Eigenschaften (PEPs). Diese Eigenschaften sind eher als Dispositionen oder auch anthropologisch fundierte Potentiale zu verstehen, denn als unveränderliche Merkmale, die das Subjekt qua Geburt besitzt. Sie können sich demnach erst in der Beziehung zu und Interaktion mit der Welt entfalten (vgl. Archer 2000: 188f.). 
"She [Archer] argues that the properties and powers of human beings are neither pre-given, nor socially bestowed, but realised through (emergent from) our practical transactions and relations with natural, practical and social environment." (Hartwig 2010: 80, A. d.V.)

Hartwig deutet hier an, wie genau subjektive Weltverortung analytisch nach Archer zu verstehen ist: Das Subjekt ist immer schon eingebettet in die Bereiche des Natürlichen, des Praktischen und des Sozialen. Der Bereich des Natürlichen umfasst all die körperlichen Beziehungen des Akteurs zu seiner Umwelt, was mit dem (somatischen) Wissen um die eigene Leiblichkeit einhergeht. Der Bereich des Praktischen konturiert einen Schnittpunkt zwischen natürlicher und sozialer Dimension und beinhaltet die gegenständlichen Beziehungen des Akteurs. Er ist durch praktisches Wissen im Umgang mit nicht-menschlichen Artefakten (material culture) gekennzeichnet. Der soziale Bereich wird durch die diskursiven Beziehungen des Akteurs gefüllt und enthält propositionales Wissen (vgl. Archer 2000: 162; Archer 2007: 8).

Der Bereich des Sozialen gleicht nahezu der von Rosa beschriebenen, horizontalen Dimension der Weltbeziehungen. Diese horizontale Dimension umfasst »die sozialen Beziehungen zu anderen Menschen, also etwa Freundschaften oder Intimbeziehungen, oder auch politische Beziehungen« (Rosa 2016: 331). Auch der Bereich des Praktischen findet bei Rosa eine Entsprechung, wenn etwa von den »Beziehungen zur Dingwelt« (ebd.: 331) oder der diagonalen Weltbeziehungsdimension die Rede ist. Einzig der Bereich des Natürlichen unterscheidet sich deutlich in seiner Extension. In den Begrifflichkeiten Rosas kommt der subjektiven Erfahrung des Überindividuellen der Welt ein wichtiger Stellenwert zu (vgl. ebd.: 331) - ein Blickwinkel auf den Archer nicht zu sprechen kommt. ${ }^{9}$

Sowohl Archer als auch Rosa konzipieren die drei Bereiche/Dimensionen als überlappend mit mäandernden Demarkationslinien (vgl. Rosa 2016: 297, insbesondere in den Ausführungen zu Ritualen; Archer 2000: 162), sodass

9 | Hier zeigt sich bei Rosa deutlich das Vermächtnis der philosophischen Anthropologie. Max Scheler konzipiert den Umgang mit der Welt als Ganzem in ganz ähnlicher Art und Weise: "Nach dieser Entdeckung der Weltkontingenz und des seltsamen Zufalls seines nun weltexzentrisch gewordenen Seinskernes war dem Menschen noch ein doppeltes Verhalten möglich: Er konnte sich darüber verwundern ( $\vartheta \alpha v \mu \alpha ́ \zeta \varepsilon \imath v)$ und seinen erkennenden Geist in Bewegung setzen, das Absolute zu erfassen und sich in es einzugliedern - das ist der Ursprung der Metaphysik jeder Art; [...] Er konnte aber auch aus dem unbezwinglichen Drang nach Bergung nicht nur seines Einzel-Seins, sondern zuvörderst seiner ganzen Gruppe, auf Grund und mit Hilfe des ungeheuren Phantasieüberschusses [...] diese Seinssphäre mit beliebigen Gestalten bevölkern, um sich in deren Macht durch Kult und Ritus hineinzubergen“ (Scheler 1991: 85, Herv. i.0.). 
die verschieden nuancierten Ausbuchstabierungen und Betonungen einzelner Elemente der Bereiche hier nicht weiter ins Gewicht fallen sollen. Gemeinsam ist beiden weltlichen Unterteilungen aber ihrer Definition nach der Anspruch auf Ausschließlichkeit. Es gibt keine (weiteren) Beziehungen oder Verortungen des Subjekts, die nicht von diesem aufgespannten Zelt der Dimensionen überdeckt werden. Ebenso verfolgen sowohl Rosa als auch Archer mit der Ausdifferenzierung ein gemeinsames Ziel: Eine realistische Anthropologie, die Subjekte nicht allein als soziale, gemeinschaftliche Wesen entwirft. Nach Archer ermöglicht es diese multiple Verortung, dass das Individuum überhaupt einen Einfluss auf die Formung des eigenen Selbst nehmen kann.

In der Subjektkonzeption Archers nehmen individuelle concerns ${ }^{10}$ der Subjekte eine bedeutende Rolle ein. Die grundlegenden concerns resultieren aus der fortwährenden Bezogenheit des Individuums auf die drei Bereiche von Welt. Sie stellen ihrerseits die Basis von Handlungsfähigkeit - im Sinne der angesprochenen PEPs - dar.

"Thus, our physical well-being depends upon establishing successful practices in the natural world; our performative competence relies upon acquiring skilful practices in relation to material artefacts; and our self-worth hinges upon developing rewarding practices in society." (Archer 2007: 8)

Konstitutiv für eine subjektiv verstandene Individualität sind folglich das Streben nach performativen Geschick und körperlichen Wohlergehen sowie der Erhalt des eigenen Selbstwertes. Die Befriedigung der grundlegenden concerns kann unterschiedliche Handlungsentwürfe und Praxen erfordern, die vom Individuum diskutiert und gewichtet werden müssen. Innerhalb dieser Abwägungs- und Tarierungsprozesse müssen jedoch stets alle Bereiche der Welt respektive die aus diesem Bezug resultierenden concerns Berücksichtigung erfahren.

Erlangen bei Rosa Emotionen eine zentrale Funktion als Indikator für die genuine Resonanzfähigkeit des Individuums ${ }^{11}$, so dienen sie bei Archer als

10 | Concern kann in der englischen Verwendung ein Interesse oder Anliegen, ebenso aber auch ein Bedürfnis oder eine Sorge beschreiben. Die Begriffsverwendung im Englischen lässt sich damit nicht eindeutig ins Deutsche übertragen, da sowohl bewusst artikulierte Ziele und Bedürfnisse als auch unbewusste Regungen gemeint sind. Im Folgenden wird deshalb der Begriff concern unübersetzt beibehalten.

11 | Wenn wir uns eine Welt (oder auch einen Menschen) ohne Lachen oder Weinen vorstellen, gewinnen wir eine ziemlich gute Vorstellung von einer - technisch, sozial, intellektuell sicher möglichen - Welt (oder einem Leben) in resonanzfreien Weltverhältnissen." (Rosa 2016: 136f.) Sehr anschaulich wird die Bedeutung von Emotionen ebenfalls in den Ausführungen zu Musik und deren Wirkung. Siehe dazu auch ebd.: $163 f$. 
>Entscheidungshilfe $<$ beim Abwegen der einzelnen concerns. Emotionen verstanden als »commentaries upon our concerns « (Archer 2000: 195) ermöglichen so eine - nicht nur rationale - Gewichtung und Beurteilung verschiedener Handlungsalternativen. Sie geben dem Akteur Auskunft darüber, wie ein entworfener Handlungsplan sich >anfühlen könnte. Aufbauend auf dieser Basis konzipiert Archer die Subjektbildung als Entwicklungsprozess, der die gängige, auch von Rosa kritisierte Natur-Kultur-Dichotomie unterläuft.

Zunächst verfügt der Akteur über einen subjektiven Selbstsinn, der sich klar von einem sprachlich-grammatikalischen Konzept des Selbst abgrenzt. Der subjektive Selbstsinn wird als vorpersonales Bewusstsein des eigenen Selbst verstanden und rührt aus der Abgrenzung des eigenen Körpers von der ihn umgebenden (Um-)Welt her (vgl. ebd.: 130). ${ }^{12}$ Der subjektive Selbstsinn ist wiederrum die Basis für den Akteur sich als in die Welt gestellt zu erfahren. Archer buchstabiert die Verortung des Akteurs und die damit einhergehenden Einschränkungen und Ermöglichungen systematisch aus. Der Akteur erwirbt seine soziale Identität im Durchlaufen verschiedener Entwicklungsinstanzen: primary agents, corporate agents und actor. Primary agents bezeichnet die soziale Positionierung des Akteurs im Moment des Zur-Welt-Kommens, die Archer mit den Adverbien »involuntarily, unconsciously, but inexorably« (Archer 2000: 135) fasst. Diese Positionierung setzt dem Akteur bereits Grenzen und schreibt ihm spezifische Merkmale zu (z.B. Muttersprache). In diesem Stadium bildet sich das qua Menschsein vorhandene Potential zur Reflexion zur konkreten, nutzbaren Fähigkeit aus (vgl. ebd.: 263).

Als Kollektivakteur (corporate agents) beginnt das Individuum sich als zugehörig zu Gruppen mit gemeinsamen Merkmalen zu begreifen, indem es ein Wir-Gefühl entwickelt und lernt, kollektive Interessen $\mathrm{zu}$ formulieren und aktiv zu verteidigen. Rosa macht einen ähnlichen Punkt stark, wenn er auf die Arbeiten von Michael Tomasello verweist (vgl. Rosa 2016: 372), welcher eine

12 | "The human body is unique, because of its dual role as the sources of perception which is also able to sense itself. It is particularly in touching oneself, where there is only one sensation unlike touching a table when toucher and touched are distinct, that the self-consciousness which constitutes me as subject, rather than object, arises. Touched objects are thus established as not being part of myself, whereas in touching my own body I have a sensory experience which demarcates me from the rest of the world." (Archer 2000: 130) Archer gründet ihre Überlegungen an dieser Stelle auf die theoretischen Ansätzen Maurice Merleau-Pontys, auf welche auch Rosa mehrfach verweist (vgl. bspw. Rosa 2016: 85). Bezeichnenderweise findet sich bei allen dreien eine sehr dichte Beschreibung eines ursprünglich nicht-kognitiven, auf unmittelbare somatische Erfahrungen beruhenden Weltbezugs. Interpretative und reflexive Fähigkeiten sind dann, obwohl bereits dispositional angelegt, ein zeitlich nachgeordnetes Entwicklungsprodukt. 
shared intentionality und eine sich durch Perspektivübernahme einstellende we-ness als Grundstein menschlich kooperativer Interaktion ansieht (vgl. Tomasello 2009).

Aufgrund der Verortung in einer konkreten, historischen Gesellschaft verfügt der actor über ein Set an Rollen, welche für ihn potenziell besetzbar sind. Rollen sind, in Abgrenzung zu Goffmans Rollenbegriff, als elastisch zu verstehen, weil sie zwingend einer individuellen Personifizierung innerhalb eines strukturell vorgegebenen Rahmens bedürfen (vgl. Waldenburger 2014: 287). Der actor wählt die Rollen selbst, die er übernehmen möchte und verwandelt sich - um mit Rosas Begrifflichkeiten zu sprechen - im Prozess der Rollenausübung die Welt an.

Aus den drei bis hierhin besprochenen Elementen bildet sich, so Archer, die soziale Identität des Individuums, welche gemeinsam mit der personalen Identität das Selbst als handlungsfähiges Subjekt konstituiert. Hier lässt sich das von Rosa verwendete Konzept der Kognitiven Landkarten als Vergleichsfolie heranziehen. Solche Landkarten zeigen dem Subjekt »was es gibt in der Welt und wie die Welt beschaffen ist« (Rosa 2016: 216). Mit anderen Worten: Die kognitiven Landkarten zeigen dem Subjekt genau die strukturellen Einschränkungen und Ermöglichungen auf, die es qua seiner immerwährenden Welt-Verortung erfährt. Strukturelle Einflüsse konditionieren, determinieren jedoch nicht. Rosa komplettiert seine Konzeption der Landkarten mit der Ergänzung, dass diese Landkarten »aber auch [aufzeigen]: worauf es dabei (für die Subjekte) jeweils ankommt, welche Haltung die jeweils richtige ist« (ebd.: 216, A. d.V.).

Die subjektiv wertende Dimension ergibt sich bei Archer aus dem Zusammenwirken der sozialen und personalen Identität im Rahmen der inneren Konversation. Die innere Konversation ist einerseits eine Analyse des sozialen Selbst über die Potenziale und Verpflichtungen, die mit der sozialen Verortung und der Besetzung von Rollen einhergehen. Anderseits setzt das Subjekt in diesem Gespräch das eigene soziale Selbst mit den concerns aus den Bereichen des Natürlichen und des Gegenständlichen ins Verhältnis. Hierbei müssen, wie bereits weiter oben erläutert, alle grundlegenden concerns Berücksichtigung finden. Die Kernkompetenz der inneren Konversation ist die Fähigkeit zur Reflexivität. Mit dieser Fähigkeit wägt das Subjekt die Anliegen und dazugehörigen Emotionen gegeneinander ab, handelt Interessen aus und fasst abschließend eine prospektive Handlungsweise ins Auge. Dieser innere Prozess wird von Archer als Dialog konzipiert, indem das Selbst mit seinem zukünftigen Selbst diskutiert (vgl. Archer 2003: 57-78). Das (immer nur vorläufige) Ergebnis der inneren Konversation bezeichnet Archer als ultimate concerns, das heißt, jenes worauf es für das Subjekt letztlich ankommt. Die ultimate concerns müssen subjektiv lebenswert und in ihrer Ausführung objektiv funktionstüchtig sein (vgl. ebd.: 14). Sie setzen demnach nicht nur jenes, wonach das Subjekt 
strebt, sondern legen auch einen Pfad fest, wie es dazu kommt und wie sich alle anderen Anliegen in Relation zu diesen ultimate concerns verhalten.

Die Resonanz- bzw. die Entfremdungskategorie Rosas sind subjektiv gewendet an dieser Stelle unter Umständen in zweifacher, analytischer Weise anschlussfähig. Zum einen könnte man Resonanz als einen spezifischen ultimate concern des Subjekts verstehen. Dies würde jedoch bedeuten, dass die Bejahung von resonanten oder zumindest als resonant wahrgenommenen Weltbeziehungen in einer heterogenen Welt nur ein möglicher Modus von vielen ist. Vor allem ein analytisch gleichwertiger Modus unter vielen. Man müsste dann zur Beurteilung ein externes, normatives Kriterium anlegen, um zu zeigen, warum dieser Modus a priori besser ist als andere. Zum anderen wäre es möglich, Resonanz als ein subjektives Passungsverhältnis zwischen der Formulierung der ultimate concerns und deren Umsetzung zu recodieren. Die Welt würde dann als antwortend erscheinen, je nachdem wie >erfolgreich< die Verwirklichung der eigenen ultimate concerns - der modus vivendi - ist (vgl. Archer 2000: 221). ${ }^{13}$ Auch in letzterer Variante bleibt allerdings ohne zusätzliches Kriterium offen, welche Art der Lebensführung - als modus vivendi und Weltanverwandlung normativ erstrebenswert ist. Im Gegensatz zu Rosas Ausführungen (vgl. Rosa 2016: 27-34 - Geschichte von Adrian und Dorian) ließe sich hier nämlich nicht so einfach eine instrumentelle Weltbeziehung als zwangsläufig nicht-responsiv respektive entfremdet kennzeichnen.

Aus unserer Sicht spricht in der Summe einiges dafür, eine gewisse konzeptionelle Nähe zwischen der Subjektkonzeption Archers und Rosas Ausführungen zu konstatieren. Allerdings betrifft diese Nähe gerade nicht die für Rosa so zentralen, normativen Aspekte des Entfremdungs- wie Resonanzbegriffes.

\section{FAZIT}

Wir haben im Rahmen des Beitrages versucht, die sozialtheoretische Kompatibilität von Rosas Soziologie der Weltbeziehung mit den entsprechenden Standpunkten des CR abzuklopfen. Dabei lässt sich zunächst festhalten, dass beide Perspektiven von einer Auseinandersetzung mit Konzepten des jeweils anderen Ansatzes profitieren können. Für den CR scheinen beispielsweise eine weiterführende Betrachtung des Konzepts der Weltbeziehung und die Ausbuchstabierung der Weltverortung des Subjektes äußerst fruchtbare Unter-

13 | Analog dazu beschreibt Entfremdung dann das Scheitern der Umsetzung der ultimate concerns. Auch Archer hat sich mit dem Scheitern der Umsetzung beschäftigt und inn in Anschluss an Sennet als driften gelabelt (vgl. Archer 2000: 246-249). 
fangen darzustellen. ${ }^{14}$ Die Soziologie der Weltbeziehung würde aus unserer Sicht ihrerseits vor allem durch eine begrifflich schärfer gefasste Sozialtheorie gewinnen.

Deutlich ambivalent fällt das Fazit hinsichtlich der bei Rosa zentralen Resonanz- bzw. Entfremdungsbegriffe aus. Zum einen erscheint ihre Konzeption selbst in Rosas Theorieentwurf nicht abschließend konsistent - was auch an der relativ offen gehaltenen sozialtheoretischen Einbettung liegt - und zum anderen ist zumindest diskutabel, ob sie auf den hier diskutierten Ebenen einen analytischen Mehrwert besitzen. Einzig und allein eine größere, theoretische Reformulierung - beispielsweise die im Beitrag vorgeschlagene subjektive Wendung - könnte aus der Sicht des CR eine produktive Integration gewährleisten.

\section{LITERATUR}

Archer, Margaret Scotford (1995): Realist Social Theory. The Morphogenetic Approach, Cambridge/New York: Cambridge University Press.

Archer, Margaret Scotford (2000): Being Human. The Problem of Agency, Cambridge/New York: Cambridge University Press.

Archer, Margaret Scotford (2003): Structure, Agency, and the Internal Conversation, Cambridge/New York: Cambridge University Press.

Archer, Margaret Scotford (2007): Making our Way Through the World. Human Reflexivity and Social Mobility, Cambridge: Cambridge University Press.

Archer, Margaret Scotford (Hg.) (2010): Conversations About Reflexivity, London/New York: Routledge.

Archer, Margaret Scotford (2010): »Can Reflexivity and Habitus Work in Tandem? «, in: Dies. (Hg.), Conversations About Reflexivity, London/New York: Routledge, S. 123-143.

Bhaskar, Roy (1998): The Possibility of Naturalism. A Philosophical Critique of the Contemporary Human Sciences, London/New York: Routledge.

Bhaskar, Roy (2008): A Realist Theory of Science, London: Verso.

Bhaskar, Roy (2009): Scientific Realism and Human Emancipation, London: Verso.

Bhaskar, Roy (1983): »Beef, Structure and Place«, in: Journal of the Theory of Social Behaviour 13 (1), S. 81-96.

14 | Insbesondere Sayers (2011) Ausführungen in Why things matter to people scheinen hierfür eine hervorragende Grundlage zu bieten. 
Callon, Michel (1984): »Some Elements of a Sociology of Translation: Domestication of the Scallops and the Fishermen of St. Brieuc Bay«, in: The Sociological Review 32 (1), S. 196-233.

Collier, Andrew (1994): Critical Realism. An Introduction to Roy Bhaskar's Philosophy, London/New York: Verso.

Dörre, Klaus/Lessenich, Stephan/Rosa, Hartmut (2009): »Soziologie - Kapitalismus - Kritik: Zur Wiederbelebung einer Wahlverwandtschaft«, in: Dies., Soziologie - Kapitalismus - Kritik. Eine Debatte, Frankfurt a.M.: Suhrkamp, S. 9-18.

Elder-Vass, Dave (2007): »Reconciling Archer and Bourdieu in an Emergentist Theory of Action«, in: Sociological Theory 25 (4), S. 325-346.

Elder-Vass, Dave (2008): »Searching for Realism, Structure and Agency in Actor Network Theory«, in: The British Journal of Sociology 59 (3), S. 455-473.

Fleetwood, Steve (2008): »Structure, Institutions, Agency. Habit and Reflexive Deliberations«, in: Journal of Institutional Economics 4 (2), S. 183-203.

Hartwig, Mervyn (2010): The Formation of Critical Realism. Mervyn Hartwig Interviews Roy Bhaskar, London/New York: Routledge.

Latour, Bruno (1991): We Have Never Been Modern, Cambridge/Mass.: Harvard University Press.

Latour, Bruno (1996): Aramis, or, The Love of Technology, Cambridge/Mass.: Harvard University Press.

Latour, Bruno (2005): Reassembling the Social. An Introduction to Actor-Network-Theory, Oxford/New York: Oxford University Press.

Lindner, Urs (2014): »Soziale Strukturen, generative Mechanismen und das gute Leben. Critical Realism als Philosophie der Sozialwissenschaften«, in: Zeitschrift für kritische Sozialtheorie und Philosophie 1 (2), S. 198-275.

Rosa, Hartmut (2012): Weltbeziehungen im Zeitalter der Beschleunigung. Umrisse einer neuen Gesellschaftskritik, Berlin: Suhrkamp.

Rosa, Hartmut (2016): Resonanz. Eine Soziologie der Weltbeziehung, Berlin: Suhrkamp.

Sayer, R. Andrew (2011): Why Things Matter to People. Social Science, Values and Ethical Life. Cambridge/New York: Cambridge University Press.

Sayer, R. Andrew (2010): »Reflexivity and the Habitus? « in: Margaret Scotford Archer (Hg.), Conversations About Reflexivity, S. 123-143.

Scheler, Max (1991): Die Stellung des Menschen im Kosmos, Bonn: Bouvier.

Schulz, Peter (2015): »Kritik woran? Zur Ambivalenz der kritischen Soziologie Hartmut Rosas«, in: Zeitschrift für kritische Sozialtheorie und Philosophie 2 (1), S. 101-117.

Tomasello, Michael (2009): Why We Cooperate, Cambridge/Mass.: MIT Press.

Waldenburger, Lisa (2014): »Situierte Reflexivität. Margaret Archers Entwurf eines kritisch-realistischen Subjektverständnisses«, in: Zeitschrift für kritische Sozialtheorie und Philosophie 1 (2), S. 276-297. 